\title{
Review Article \\ Pathogenesis of Bone Alterations in Gaucher Disease: The Role of Immune System
}

\author{
Juan Marcos Mucci and Paula Rozenfeld \\ IIFP, Departamento de Ciencias Biológicas, Facultad de Ciencias Exactas, Universidad Nacional de La Plata y CONICET, \\ 47 y 115, 1900 La Plata, Argentina
}

Correspondence should be addressed to Paula Rozenfeld; paurozen@biol.unlp.edu.ar

Received 8 October 2014; Revised 9 January 2015; Accepted 11 January 2015

Academic Editor: Giacomina Brunetti

Copyright (C) 2015 J. M. Mucci and P. Rozenfeld. This is an open access article distributed under the Creative Commons Attribution License, which permits unrestricted use, distribution, and reproduction in any medium, provided the original work is properly cited.

Gaucher, the most prevalent lysosomal disorder, is an autosomal recessive inherited disorder due to a deficiency of glucocerebrosidase. Glucocerebrosidase deficiency leads to the accumulation of glucosylceramide primarily in cells of mononuclear-macrophage lineage. Clinical alterations are visceral, hematological, and skeletal. Bone disorder in Gaucher disease produces defects on bone metabolism and structure and patients suffer from bone pain and crisis. Skeletal problems include osteopenia, osteoporosis, osteolytic lesions, and osteonecrosis. On the other hand a chronic stimulation of the immune system is a well-accepted hallmark in this disease. In this review we summarize the latest findings in the mechanisms leading to the bone pathology in Gaucher disease in relationship with the proinflammatory state.

\section{Osteoimmunology}

A diverse number of interactions between bone and immune cells occur within the bone microenvironment. Bone and immune cells share the same progenitors residing in the bone marrow and these progenitors are under the effect of the same molecules including cytokines; these molecules can have a high influence in the hematopoiesis process, local immune responses, and bone cell development.

There is evidence that several immune cells can influence bone cell development and activity. However, the key players in this regulation are activated T-cells. After successful antigen-specific activation, T-cells produce a number of proinflammatory cytokines [1] that can act directly or indirectly on cells involved in bone turnover shifting bone balance towards bone resorption or bone generation.

The bone turnover process involves bone removal by resorbing osteoclasts and bone formation by osteoblasts. These processes are strictly regulated in physiological conditions, and this regulation implies the participation of osteocytes, which are the final step of osteoblast differentiation [2].
Osteoclasts are bone resorbing cells that derive from the same progenitors as macrophages and dendritic cells (monocyte/macrophage lineage) [3]. RANKL and macrophage colony stimulating factor (M-CSF) are essential for commitment of the common precursor to the osteoclast lineage and survival of differentiated osteoclasts. In addition numerous cytokines are also able to influence osteoclast differentiation and/or function [4].

Osteoblasts are the bone forming cells that originate from bone marrow-residing multipotent mesenchymal stem cells. Osteoblasts are one of the major sources of RANKL and in this manner they control bone resorption. These cells can influence immune cells and are critical regulators of the hematopoietic stem cells (HSC) from where immune and other blood cells derive [5].

RANKL is a transmembrane protein of the TNF superfamily encoded by the Tnfsfl1 gene. It is expressed on the surface of osteoblasts (at different stages of differentiation), osteocytes, stromal cells of undefined origin, B- and T-cells, synovial fibroblasts, hypertrophic chondrocytes, and even osteoclasts themselves. The receptor of RANKL is RANK, 
which is encoded by the Tnfrsf11a gene. Upon stimulation of RANK by RANKL under costimulatory signals such as MCSF, the process of osteoclast differentiation and maturation begins [6]. The third protein member of the osteoclastogenesis axis is called osteoprotegerin (OPG) and is encoded by the Tnfrsfl1b gene. OPG functions as a soluble decoy receptor for RANKL, inhibiting RANKL interaction with RANK, thus acting like an antiosteoclastogenic molecule [7]. OPG is expressed by osteoblasts and other mesenchymal cells [8].

The RANK/RANKL/OPG axis is essential in osteoclast differentiation in vivo as mutations in genes encoding RANKL, RANK, or OPG lead to disorders with high bone pathology [9]. RANKL is presented in two different forms as a membrane-anchored molecule or as a soluble protein released by the action of matrix metalloproteinases [10]. Both forms of the protein have osteoclastogenesis activity; however, the membrane-anchored form functions more efficiently [11].

The expression of RANKL on mesenchymal cells, such as osteoblasts, is upregulated by osteoclastogenic factors such as vitamin D3, prostaglandin E2, parathyroid hormone, and several cytokines including IL-1, IL-6, IL-11, IL-17, and TNF- $\alpha$ [12].

\section{Osteoimmunology in Pathological Conditions}

The activation of immune cells is a requisite for defense of the host against pathogens; however, a persistent overactivation of effector cells under certain pathological conditions can result in tissue damage.

In the early 1980s, osteoclasts were identified throughout the synovium and at the synovium/bone interface in joints of rheumatoid arthritis (RA) patients [13]. These observations led to the determination that osteoclasts play an important role in certain pathological conditions $[14,15]$.

Inflammatory cytokines such as IL-1, IL-6, and TNF- $\alpha$ are present at high levels in the synovial fluid and synovium of RA patients. These cytokines have a potent capacity to induce the expression of RANKL on synovial fibroblasts and bone derived stromal cells and to affect osteoclast differentiation, thus directly contributing to the bone destruction process [13].

Osteoporosis (OP) has been traditionally considered as an endocrine disease resulting mainly from the estrogens decline after menopause. This change affects bone remodeling, leading to higher risk of fractures. Since the endocrine point of view by itself does not completely explain the pathogenesis of OP, the osteoimmunological approach raised and suggested that the production of proinflammatory cytokines such as TNF- $\alpha$, IL-1, IL- 6 , IL-7, and IFN- $\gamma$ by activated $\mathrm{T}$ lymphocytes could contribute to menopausal changes in bone dynamics $[16,17]$.

Phenylketonuria (PKU) is an inborn error of amino acid metabolism resulting from deficiency of phenylalanine hydroxylase, the key enzyme for phenylalanine metabolism. Bone impairment has been widely documented in PKU, using both radiological and ultrasound methods [18, 19], and it is typically associated with increasing age. In 2010 it was shown that PKU patients present increased numbers of circulating osteoclast precursors with higher differentiation potential compared to healthy controls. TNF- $\alpha$ levels and the RANKL/OPG ratio were increased in supernatants of PBMC cultures from patients and it was shown that the increased osteoclast differentiation from PBMC was RANKL dependent [20].

\section{Gaucher Disease}

Gaucher disease is the most prevalent lysosomal disorder [21], of around 1:13,000-60,000, and with a higher frequency in the Ashkenazi Jewish population [22]. Gaucher disease (GD; MIM \#230800) is an autosomal recessive inherited lysosomal storage disorder that is due to a deficiency of glucocerebrosidase (acid beta glucosidase; GCase; EC 3.2.1.45). GCase deficiency results in progressive, intralysosomal accumulation of glucosylceramide in different tissues, primarily in cells of mononuclear-macrophage lineage. Lipid accumulation in macrophages results in engorged cells called "Gaucher cells." Rarely, a variant GD may be secondarily caused by deficiency of the saposin C, the activator of the enzyme [23].

The first specific treatment for a lysosomal disorder was introduced for Gaucher disease, the enzyme replacement therapy (ERT) [24].

Clinical phenotype of GD reflects a continuum ranging from neuronopathic forms (GD types II and III) to the more frequent visceral form (GD type I) and from early onset to late onset [25].

Type I is observed in $90 \%$ of cases and is characterized by the lack of CNS manifestations. Clinical alterations are visceral (hepatosplenomegaly without organ disfunction), hematological (anemia and thrombopenia), and skeletal [26].

Bone pathology remains the main problem for GD I patients after the introduction of enzyme replacement therapy. Bone disease is a common and often painful and disabling manifestation of GD. Multiple compartments of bone that are affected are caused by alterations in bone metabolism (turnover, remodeling, and mineralization).

Almost all GD patients develop skeletal complications, consisting mainly of remodeling failure, osteopenia, osteoporosis, marrow infiltration, avascular necrosis, and osteolysis [27]. It may be suggested that patients with early onset GD I are at risk of skeletal disease. One of the early signs is the typical "Erlenmeyer flask" deformity of the distal femur. These changes predominantly affect long bones and the vertebrae. Patients could be asymptomatic with or without radiological signs or present symptoms including bone pain involving one limb or joint, avascular necrosis, or pathological fractures. An international registry of Gaucher patients worldwide revealed that $62 \%$ of them had some form of radiologic bone disease and $43 \%$ experienced bone pain [28]. The $M \phi$ are prominent in the bone marrow and contribute to acute episodes of osteonecrosis, particularly during growth. Necrosis of the marrow leads to impaired 
function of joints. Other effects on the skeleton include local swellings known as Gaucheromas.

Imaging methodologies for the evaluation of skeletal involvement, such as conventional (plain) radiography and scintigraphy, MRI, computed tomography, or dual energy $\mathrm{X}$-ray absorptiometry, are currently employed and provide accurate evaluation and staging of bone lesions in GD [29].

Much evidence demonstrates substantial improvement of hematological and visceral parameters upon introduction of specific ERT for Gaucher patients [30]. However, bone tissue does not respond equally; it is, in some degree, refractory to therapy. Patients at risk may benefit from early intervention with ERT, although many lesions and osteonecrosis are irreversible. Enzyme therapy cannot reverse established osseous injury [31]. Several prospective studies have been performed to evaluate the effectiveness of ERT in treating skeletal pathology. Bone pain is present at baseline in around two-thirds of the patients. Some patients improve in this aspect, but $40 \%$ of patients remain with this symptom after 18 months of treatment. In a recent study of patients treated with imiglucerase for 10 years, a positive effect was observed in skeletal symptoms, as well as a reduction of bone pain and crises in patients who suffer from them at baseline. Moreover, most of the patients who did not report bone symptoms at baseline continued to be pain-free after 10 years of ERT [32].

Bone mineral density tends to increase during therapy, but the response is slow [31]. Patients with preexistent skeletal complications tend to suffer incidents during ERT, such as medullary infarctions, avascular necrosis, or fractures, but the frequency of these events is reduced [33]. Low bone density manifests early in children with GD, and mineral density deficit is maximal in the adolescent period. Moreover, this group is most responsive to ERT, underscoring the importance of early diagnosis and intervention to achieve optimal peak bone mass [34]. In the largest study with treated pediatric patients bone mineral health was impaired in a large proportion of the group before ERT and improved considerably with treatment [35].

\section{Inflammation in Gaucher Disease}

A chronic stimulation of the immune system is a wellaccepted hallmark in GD. Studies of the proinflammatory state in patients were mainly focused on analyzing cytokine levels in sera [36-38]. Although there is a high variation among patients, increased levels of IL- $1 \alpha$, IL-1 $\beta$, IL-1Ra, sIL2R, IL-6, IL-8, IL-10, IL-18, TNF- $\alpha$, TGF- $\beta$, M-CSF, MIP-1, and CCL18 have been reported in sera [39-41].

Macrophages $(\mathrm{M} \phi)$ are the principal cell type compromised in patients with GD. M $\phi$ have several different functions including tissue remodeling and host defense; on the other hand they play central roles in many disease processes. They can secrete both anti- or proinflammatory cytokines depending on the activation signals. Upon activation, two main phenotypes of $\mathrm{M} \phi$ could be produced: classical or alternative, depending on environment present at the time of the stages of activation [42]. Gaucher cells resemble alternative activated $\mathrm{M} \phi$ [43], characterized by the expression of chitotriosidase and CCL18.

Several immune cells have been shown to be impaired in GD including monocytes, $\mathrm{M} \phi$, dendritic cells, and T- and B-cells [44-46]. It has been shown that monocytes in GD patients expressed higher levels of CD1d and MHCII on their surface, which could lead to an increased T-cell activation [47]. Abnormalities in the B-cell subset are mainly IgG and IgM hypergammaglobulinemia and plasmacytosis [48]. An increased incidence of gammopathies and multiple myeloma has been reported, further showing the interplay between Gaucher cells and the immune system [49].

A GD murine model was generated, in which the $G B A$ gene was conditionally deleted on hematopoietic cells [50]. This model presented all the hallmark characteristics of GD I, including organomegaly, and it was the only murine model so far to show bone involvement. In this model an alteration of immune cell compartment was observed. This alteration included thymic maturation impairment with higher levels of $\mathrm{CD}^{+}$and antigen-presenting cells. What is more, activated B-cells on the thymus were also increased, which could explain the alteration of normal T-cell maturation [51].

In another murine model of GD, higher levels of $\mathrm{CD} 4^{+}$ cells were found on the lungs, spleen, and liver as well as an increased expression of costimulatory molecules [52]. Higher levels of proinflammatory cytokines including IFN- $\gamma$, IL$12 \mathrm{p} 40$, TNF- $\alpha$, IL-17A/F, IL- 6 , and TGF- $\beta$ were found in sera of these mice. When T-cells were cocultured with dendritic cells in the presence of glucosylceramide, higher levels of Th1/Th17 cytokines were secreted.

More recently, using a different approach, Panicker et al. differentiated $\mathrm{M} \phi$ from patient induced pluripotent stem cells (hiPSC); with this model they showed increased production of IL- $1 \beta$, TNF- $\alpha$, and IL- 6 by GD derived $\mathrm{M} \phi$ and an exacerbated response to LPS treatment [53].

This deregulation of immune system cells is tightly related to the increased levels of cytokines and chemokines. These molecules are secreted by the immune cells, which, in turn, are recruited and activated by chemokines and cytokines, respectively. This could create a loop in which immune cells from Gaucher patients are being continuously activated, leading to systemic and focal activation of the immune system.

\section{Osteoclast-Osteoblast Uncoupling in Gaucher Disease}

The molecular and cellular bases of GD bone physiopathology are not well understood and opposing studies have emerged in the last few years. As mentioned before in 2010, Mistry et al. [50] generated a conditional KO mouse model of GD I which presented the main GD clinical hallmarks. The most striking feature about this model is the presence of bone involvement as previous mouse models of GD did not present bone involvement. Bone manifestations included medullar infarctions with associated avascular necrosis and osteopenia at all sites. The bone formation rate presented 
a significant impairment in these mice while the quantification of TRAP-labeled surfaces did not present differences.

A significant impairment in osteoblast proliferation and differentiation was present in the model, while osteoclast differentiation and activity did not seem to be altered. The impairment on osteoblast proliferation was shown to be dependent on a decrease in PKC activity due to the accumulation of glucosylsphingosine and, to a lesser extent, glucosylceramide. More recent studies present sphingosine as the most probable candidate for osteoblast impairment in the mouse model [54]. These findings suggest that bone complications in GD would result from an osteoblast source without osteoclast involvement $[50,54]$.

Different reports have shown the involvement of osteoclasts on GD bone pathophysiology. Using an in vitro model of GD in which mesenchymal stem cells and monocytes were exposed to conduritol- $\beta$-epoxide (CBE), a specific glucocerebrosidase inhibitor, Lecourt et al. showed that although direct CBE treatment had no effect on osteoclast differentiation if mesenchymal stem cells were cultured in the presence of conditioned media from CBE-exposed monocytes, an increased osteoclastogenesis and resorption activity was detected [55].

Our group showed, using a similar approach, that treatment of osteoclast precursors with conditioned media from peripheral blood mononuclear cells (PBMCs) exposed to $\mathrm{CBE}$ resulted in an increased level of osteoclast differentiation when compared to control conditioned media.

What is more, we showed that one of the central molecules involved in the increased osteoclast differentiation was the proinflammatory cytokine TNF- $\alpha$ and that T-cells also played an important role in this process [56]. The same results were obtained using a mice model in which conditioned media were obtained from peritoneal $\mathrm{M} \phi$ or splenocytes exposed to $\mathrm{CBE}$; in this model involvement of TNF- $\alpha$ was also shown using osteoclast precursors derived from TNF- $\alpha$ receptor deficient mice [57]. In addition to this we could show that treatment of the osteoblastic cell line MC3T3 with conditioned media from CBE treated $\mathrm{M} \phi$ reduced mineralization and collagen deposit [57]. These results would indicate an impairment of both osteoclast and osteoblast activity in GD leading to bone loss as the involvement of immune cells and molecules in this process.

The group of Reed et al. isolated PBMC from patients with GD and showed that patients' monocytes, when exposed to osteoclastogenic mediators, presented a higher differentiation towards active osteoclasts. What is more, osteoclasts differentiated from patients had bigger diameter and a greater number of nuclei when compared with osteoclasts differentiated from healthy controls' PBMCs. They showed that the higher osteoclastogenic potential presented a clinical correlation with patient's bone involvement [58].

\section{Future Perspectives}

GD is the most common lysosomal disorder and the first for which specific treatment has been developed. Bone disease in Gaucher patients is one of the most disabling features of the disease, so the possibility of knowing the mechanisms underlying the bone pathology is a main challenge to ameliorate the quality of life of patients.

Studies are based on the explanation of the cellular and molecular pathways that result upon glucosylceramide accumulation in $\mathrm{M} \phi$ and the possible relationship with different bone cells.

The bases of osteoimmunology are being applied to bring light in this aspect. In this regard, there are several questions to be answered. T-cell involvement and a better understanding of the effects and importance of proinflammatory cytokines such as TNF- $\alpha$ on bone pathology in GD are necessary.

On the other hand, crosstalk between osteoblasts and osteoclasts in GD could provide new mechanisms involved in the process of bone loss.

Finally the effect of ERT and substrate reduction therapy on bone involvement is a central aspect to be studied, especially, how these treatments affect different bone cells and their function.

The results of basic research will be of utility in order to identify new targets for coadjuvant therapies to treat skeletal pathology in GD.

\section{Conflict of Interests}

The authors declare that there is no conflict of interests regarding the publication of this paper.

\section{References}

[1] P. J. Delves and I. M. Roitt, "The immune system. First of two parts," The New England Journal of Medicine, vol. 343, no. 1, pp. 37-49, 2000.

[2] J. Xiong and C. A. O’Brien, "Osteocyte RANKL: new insights into the control of bone remodeling," Journal of Bone and Mineral Research, vol. 27, no. 3, pp. 499-505, 2012.

[3] T. J. de Vries, T. Schoenmaker, B. Hooibrink, P. J. M. Leenen, and V. Everts, "Myeloid blasts are the mouse bone marrow cells prone to differentiate into osteoclasts," Journal of Leukocyte Biology, vol. 85, no. 6, pp. 919-927, 2009.

[4] H. Takayanagi, K. Sato, A. Takaoka, and T. Taniguchi, "Interplay between interferon and other cytokine systems in bone metabolism," Immunological Reviews, vol. 208, pp. 181-193, 2005.

[5] T. Yin and L. Li, “The stem cell niches in bone," The Journal of Clinical Investigation, vol. 116, no. 5, pp. 1195-1201, 2006.

[6] W. J. Boyle, W. S. Simonet, and D. L. Lacey, "Osteoclast differentiation and activation," Nature, vol. 423, no. 6937, pp. 337-342, 2003.

[7] W. S. Simonet, D. L. Lacey, C. R. Dunstan et al., "Osteoprotegerin: a novel secreted protein involved in the regulation of bone density," Cell, vol. 89, no. 2, pp. 309-319, 1997.

[8] R. Siddappa, H. Fernandes, J. Liu, C. van Blitterswijk, and J. de Boer, "The response of human mesenchymal stem cells to osteogenic signals and its impact on bone tissue engineering," Current Stem Cell Research and Therapy, vol. 2, no. 3, pp. 209220, 2007.

[9] A. Leibbrandt and J. M. Penninger, "RANK/RANKL: regulators of immune responses and bone physiology," Annals of the New York Academy of Sciences, vol. 1143, pp. 123-150, 2008. 
[10] F. Kanamaru, H. Iwai, T. Ikeda, A. Nakajima, I. Ishikawa, and M. Azuma, "Expression of membrane-bound and soluble receptor activator of NF-kappaB ligand (RANKL) in human T cells," Immunology Letters, vol. 94, no. 3, pp. 239-246, 2004.

[11] T. Nakashima, M. Hayashi, T. Fukunaga et al., "Evidence for osteocyte regulation of bone homeostasis through RANKL expression," Nature Medicine, vol. 17, no. 10, pp. 1231-1234, 2011.

[12] T. Nakashima, Y. Kobayashi, S. Yamasaki et al., "Protein expression and functional difference of membrane-bound and soluble receptor activator of NF- $\kappa$ B ligand: modulation of the expression by osteotropic factors and cytokines," Biochemical and Biophysical Research Communications, vol. 275, no. 3, pp. 768-775, 2000.

[13] H. Takayanagi, "Osteoimmunology: shared mechanisms and crosstalk between the immune and bone systems," Nature Reviews Immunology, vol. 7, no. 4, pp. 292-304, 2007.

[14] E. M. Gravallese, Y. Harada, J.-T. Wang, A. H. Gorn, T. S. Thornhill, and S. R. Goldring, "Identification of cell types responsible for bone resorption in rheumatoid arthritis and juvenile rheumatoid arthritis," The American Journal of Pathology, vol. 152, no. 4, pp. 943-951, 1998.

[15] H. Takayanagi, H. Oda, S. Yamamoto et al., "A new mechanism of bone destruction in rheumatoid arthritis: synovial fibroblasts induce osteoclastogenesis," Biochemical and Biophysical Research Communications, vol. 240, no. 2, pp. 279-286, 1997.

[16] R. L. Jilka, G. Hangoc, G. Girasole et al., "Increased osteoclast development after estrogen loss: mediation by interleukin-6," Science, vol. 257, no. 5066, pp. 88-91, 1992.

[17] R. Pacifici, "Estrogen deficiency, T cells and bone loss," Cellular Immunology, vol. 252, no. 1-2, pp. 68-80, 2008.

[18] L. Hillman, C. Schlotzhauer, D. Lee et al., "Decreased bone mineralization in children with phenylketonuria under treatment," European Journal of Pediatrics, vol. 155, supplement 1, pp. S148S152, 1996.

[19] M. P. A. Hoeks, M. den Heijer, and M. C. H. Janssen, "Adult issues in phenylketonuria," The Netherlands Journal of Medicine, vol. 67, no. 1, pp. 2-7, 2009.

[20] I. Roato, F. Porta, A. Mussa et al., "Bone impairment in phenylketonuria is characterized by circulating osteoclast precursors and activated T cell increase," PLoS ONE, vol. 5, no. 11, Article ID e14167, 2010.

[21] P. J. Meikle, J. J. Hopwood, A. E. Clague, and W. F. Carey, "Prevalence of lysosomal storage disorders," The Journal of the American Medical Association, vol. 281, no. 3, pp. 249-254, 1999.

[22] J. Wittmann, E. Karg, S. Turi et al., "Newborn screening for lysosomal storage disorders in Hungary," JIMD Reports, vol. 6, pp. 117-125, 2012.

[23] G. A. Grabowski, "Gaucher disease and other storage disorders," Hematology, vol. 2012, no. 1, pp. 13-18, 2012.

[24] N. W. Barton, R. O. Brady, J. M. Dambrosia et al., "Replacement therapy for inherited enzyme deficiency-macrophagetargeted glucocerebrosidase for Gaucher's disease," The New England Journal of Medicine, vol. 324, no. 21, pp. 1464-1470, 1991.

[25] P. Mistry and D. P. Germain, "Phenotype variations in Gaucher disease," La Revue de Médecine Interne, vol. 27, supplement 1, pp. S3-S10, 2006.

[26] A. Zimran, G. Altarescu, B. Rudensky, A. Abrahamov, and D. Elstein, "Survey of hematological aspects of Gaucher disease," Hematology, vol. 10, no. 2, pp. 151-156, 2005.

[27] M. Itzchaki, E. Lebel, A. Dweck et al., "Orthopedic considerations in Gaucher disease since the advent of enzyme replacement therapy," Acta Orthopaedica Scandinavica, vol. 75, no. 6, pp. 641-653, 2004.

[28] R. J. Wenstrup, M. Roca-Espiau, N. J. Weinreb, and B. Bembi, "Skeletal aspects of Gaucher disease: a review," The British Journal of Radiology, vol. 75, supplement 1, pp. A2-A12, 2002.

[29] R. Katz, T. Booth, R. Hargunani, P. Wylie, and B. Holloway, "Radiological aspects of Gaucher disease," Skeletal Radiology, vol. 40, no. 12, pp. 1505-1513, 2011.

[30] D. Elstein, A. J. Foldes, D. Zahrieh et al., "Significant and continuous improvement in bone mineral density among type 1 Gaucher disease patients treated with velaglucerase alfa: 69-month experience, including dose reduction," Blood Cells, Molecules, \& Diseases, vol. 47, no. 1, pp. 56-61, 2011.

[31] K. B. Sims, G. M. Pastores, N. J. Weinreb et al., "Improvement of bone disease by imiglucerase (Cerezyme) therapy in patients with skeletal manifestations of type 1 Gaucher disease: results of a 48-month longitudinal cohort study," Clinical Genetics, vol. 73, no. 5, pp. 430-440, 2008.

[32] N. J. Weinreb, J. Goldblatt, J. Villalobos et al., "Long-term clinical outcomes in type 1 Gaucher disease following 10 years of imiglucerase treatment," Journal of Inherited Metabolic Disease, vol. 36, no. 3, pp. 543-553, 2013.

[33] J. Stirnemann, N. Belmatoug, C. Vincent, O. Fain, B. Fantin, and F. Mentré, "Bone events and evolution of biologic markers in Gaucher disease before and during treatment," Arthritis Research and Therapy, vol. 12, no. 4, article R156, 2010.

[34] P. K. Mistry, N. J. Weinreb, P. Kaplan, J. A. Cole, A. R. Gwosdow, and T. Hangartner, "Osteopenia in Gaucher disease develops early in life: response to imiglucerase enzyme therapy in children, adolescents and adults," Blood Cells, Molecules, and Diseases, vol. 46, no. 1, pp. 66-72, 2011.

[35] H. Andersson, P. Kaplan, K. Kacena, and J. Yee, "Eight-year clinical outcomes of long-term enzyme replacement therapy for 884 children with gaucher disease type 1," Pediatrics, vol. 122, no. 6, pp. 1182-1190, 2008.

[36] V. Barak, M. Acker, B. Nisman et al., "Cytokines in Gaucher's disease," European Cytokine Network, vol. 10, no. 2, pp. 205-210, 1999.

[37] C. E. M. Hollak, L. Evers, J. M. F. G. Aerts, and M. H. J. van Oers, "Elevated levels of M-CSF, sCD14 and IL8 in type 1 Gaucher disease," Blood Cells, Molecules and Diseases, vol. 23, no. 2, pp. 201-212, 1997.

[38] H. Michelakakis, C. Spanou, A. Kondyli et al., "Plasma tumor necrosis factor-a (TNF-a) levels in Gaucher disease," Biochimica et Biophysica Acta, vol. 1317, no. 3, pp. 219-222, 1996.

[39] R. G. Boot, M. Verhoek, M. de Fost et al., "Marked elevation of the chemokine CCL18/PARC in Gaucher disease: a novel surrogate marker for assessing therapeutic intervention," Blood, vol. 103, no. 1, pp. 33-39, 2004.

[40] M. J. van Breemen, M. de Fost, J. S. A. Voerman et al., "Increased plasma macrophage inflammatory protein (MIP)-lalpha and MIP-1beta levels in type 1 Gaucher disease," Biochimica et Biophysica Acta-Molecular Basis of Disease, vol. 1772, no. 7, pp. 788-796, 2007.

[41] M. Yoshino, Y. Watanabe, Y. Tokunaga et al., "Roles of specific cytokines in bone remodeling and hematopoiesis in Gaucher disease," Pediatrics International, vol. 49, no. 6, pp. 959-965, 2007.

[42] S. Gordon and F. O. Martinez, "Alternative activation of macrophages: mechanism and functions," Immunity, vol. 32, no. 5, pp. 593-604, 2010. 
[43] L. A. Boven, M. van Meurs, R. G. Boot et al., "Gaucher cells demonstrate a distinct macrophage phenotype and resemble alternatively activated macrophages," The American Journal of Clinical Pathology, vol. 122, no. 3, pp. 359-369, 2004.

[44] C. Braudeau, J. Graveleau, M. Rimbert et al., "Altered innate function of plasmacytoid dendritic cells restored by enzyme replacement therapy in Gaucher disease," Blood Cells, Molecules, and Diseases, vol. 50, no. 4, pp. 281-288, 2013.

[45] F. Camou and J.-F. Viallard, "Extended remission of B-cell lymphoma with monoclonal gammopathy in a patient with type 1 Gaucher disease treated with enzyme replacement therapy," Blood Cells, Molecules, and Diseases, vol. 48, no. 1, pp. 51-52, 2012.

[46] L. Lacerda, F. A. Arosa, R. Lacerda et al., "T cell numbers relate to bone involvement in Gaucher disease," Blood Cells, Molecules, and Diseases, vol. 25, no. 2, pp. 130-138, 1999.

[47] A. Balreira, L. Lacerda, C. Sá Miranda, and F. A. Arosa, "Evidence for a link between sphingolipid metabolism and expression of CD1d and MHC-class II: monocytes from Gaucher disease patients as a model," British Journal of Haematology, vol. 129, no. 5, pp. 667-676, 2005.

[48] G. E. Marti, E. T. Ryan, N. M. Papadopoulos et al., "Polyclonal B-cell lymphocytosis and hypergammaglobulinemia in patients with Gaucher disease," American Journal of Hematology, vol. 29, no. 4, pp. 189-194, 1988.

[49] T. H. Taddei, K. A. Kacena, M. Yang et al., "The underrecognized progressive nature of N370S Gaucher disease and assessment of cancer risk in 403 patients," American Journal of Hematology, vol. 84, no. 4, pp. 208-214, 2009.

[50] P. K. Mistry, J. Liu, M. Yang et al., "Glucocerebrosidase genedeficient mouse recapitulates Gaucher disease displaying cellular and molecular dysregulation beyond the macrophage," Proceedings of the National Academy of Sciences of the United States of America, vol. 107, no. 45, pp. 19473-19478, 2010.

[51] J. Liu, S. Halene, M. Yang et al., "Gaucher disease gene GBA functions in immune regulation," Proceedings of the National Academy of Sciences of the United States of America, vol. 109, no. 25, pp. 10018-10023, 2012.

[52] M. K. Pandey, R. Rani, W. Zhang, K. Setchell, and G. A. Grabowski, "Immunological cell type characterization and Th1-Th17 cytokine production in a mouse model of Gaucher disease," Molecular Genetics and Metabolism, vol. 106, no. 3, pp. 310-322, 2012.

[53] L. M. Panicker, D. Miller, O. Awad et al., "Gaucher iPSC-derived macrophages produce elevated levels of inflammatory mediators and serve as a new platform for therapeutic development," Stem Cells (Dayton, Ohio), vol. 32, no. 9, pp. 2338-2349, 2014.

[54] P. K. Mistry, J. Liu, L. Sun et al., "Glucocerebrosidase 2 gene deletion rescues type 1 Gaucher disease," Proceedings of the National Academy of Sciences of the United States of America, vol. 111, no. 13, pp. 4934-4939, 2014.

[55] S. Lecourt, V. Vanneaux, A. Cras et al., "Bone marrow microenvironment in an in vitro model of gaucher disease: consequences of glucocerebrosidase deficiency," Stem Cells and Development, vol. 21, no. 2, pp. 239-248, 2012.

[56] J. M. Mucci, R. Scian, P. N. De Francesco et al., "Induction of osteoclastogenesis in an in vitro model of Gaucher disease is mediated by T cells via TNF- $\alpha$," Gene, vol. 509, no. 1, pp. 51-59, 2012.

[57] J. M. Mucci, F. Suqueli García, P. N. de Francesco et al., "Uncoupling of osteoblast-osteoclast regulation in a chemical murine model of gaucher disease," Gene, vol. 532, no. 2, pp. 186191, 2013.

[58] M. Reed, R. J. Baker, A. B. Mehta, and D. A. Hughes, "Enhanced differentiation of osteoclasts from mononuclear precursors in patients with Gaucher disease," Blood Cells, Molecules, and Diseases, vol. 51, no. 3, pp. 185-194, 2013. 


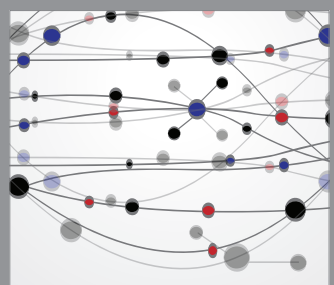

The Scientific World Journal
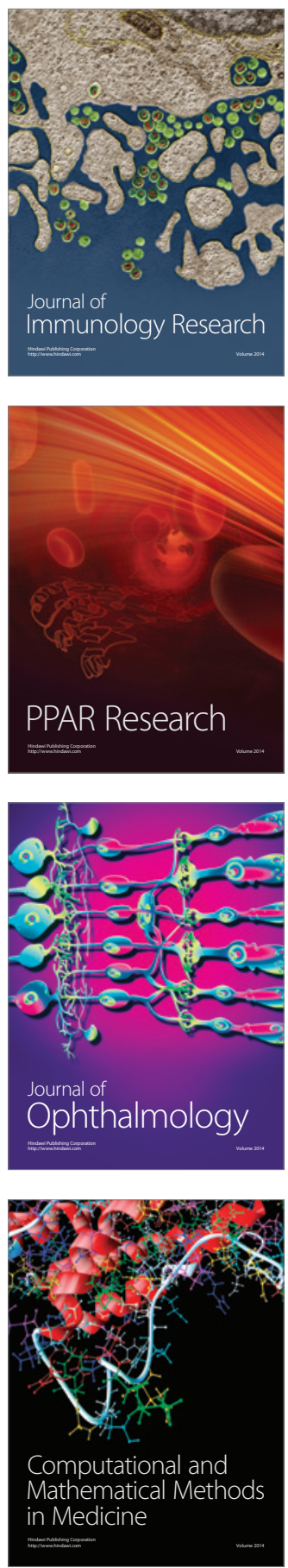

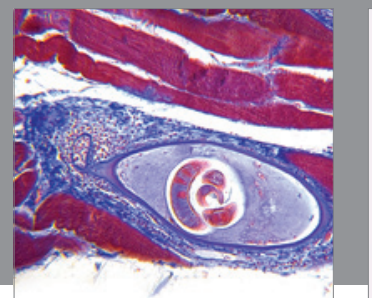

Gastroenterology

Research and Practice
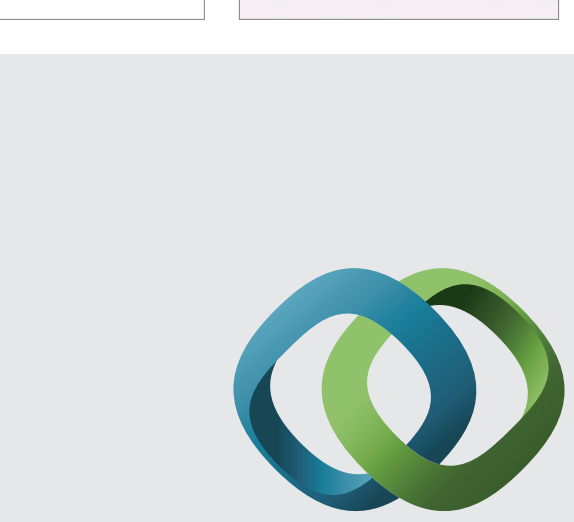

\section{Hindawi}

Submit your manuscripts at

http://www.hindawi.com
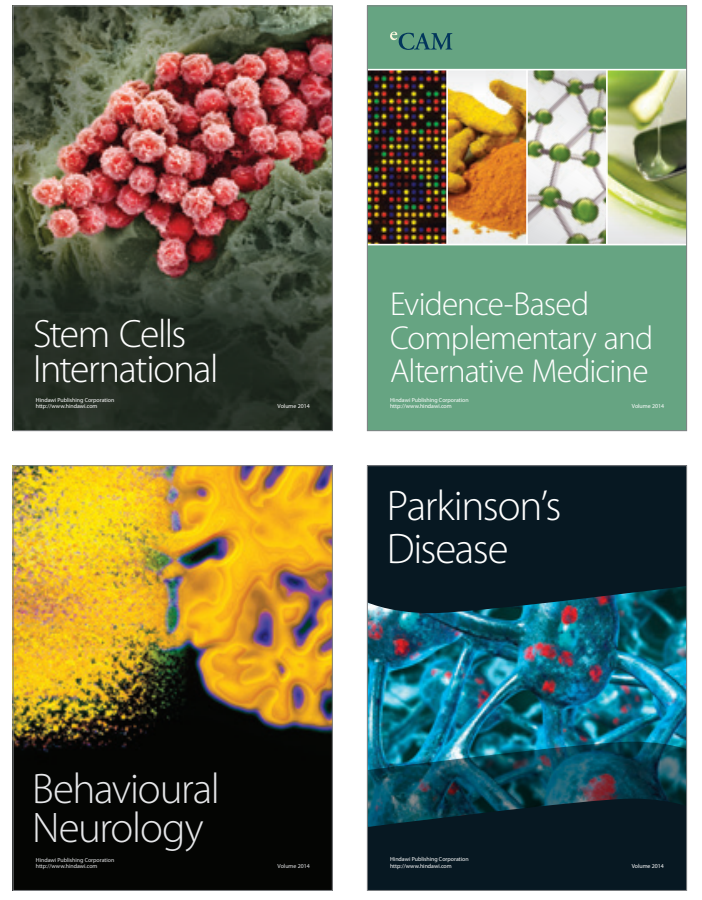
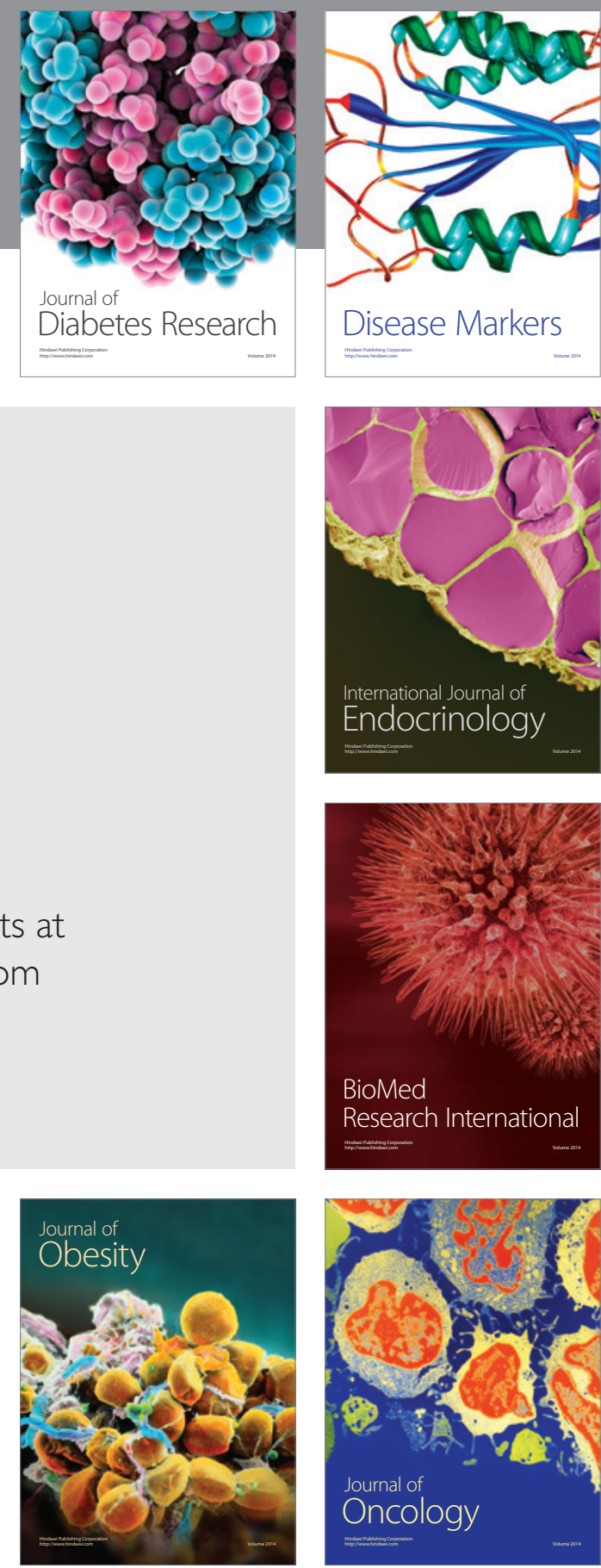

Disease Markers
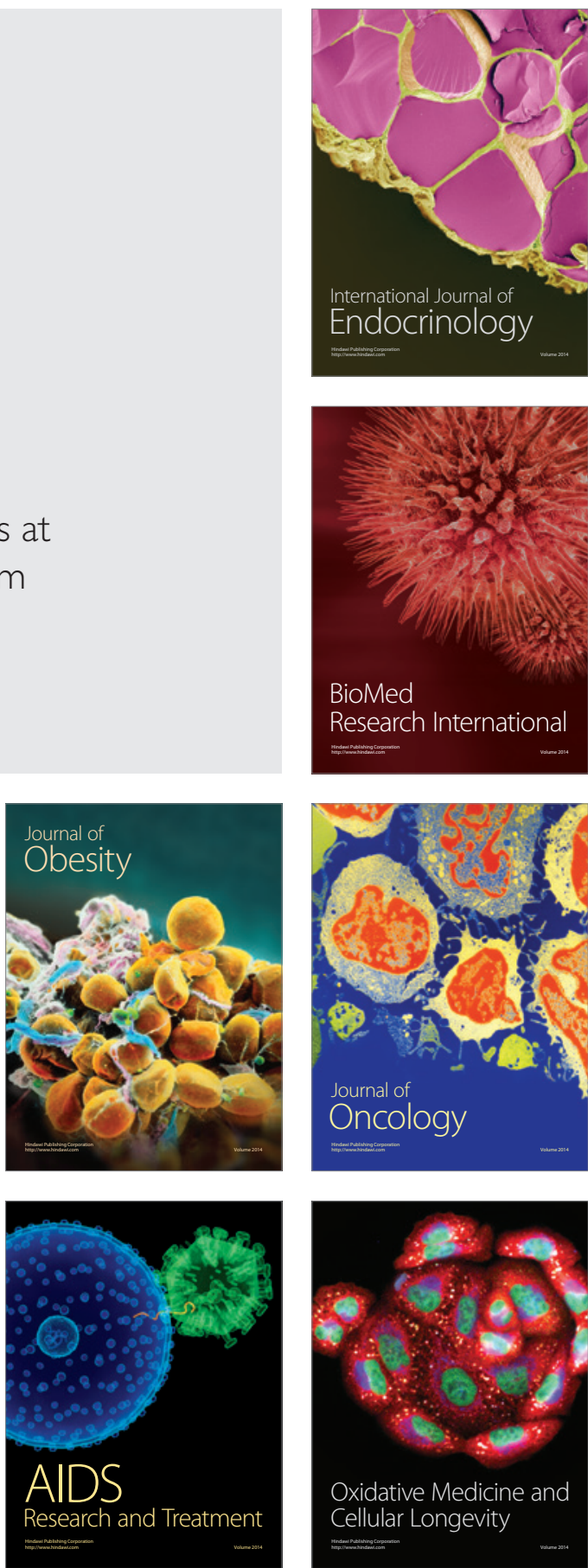